\title{
COMMISSION 19: EARTH ROTATION (ROTATION DE LE TERRE)
}

President: B.Kolaczek

Vice-president: J.Vondrak

\author{
Organizing Committee: \\ N.Capitaine \\ J.O.Dickey \\ S.Dickman \\ M.Feissel-past \\ president
}

\author{
Jin Wen-Jing \\ D.D.McCarthy \\ W.G.Melbourne \\ N.T.Mironov
}

\author{
L.Morrison \\ D. Robertson \\ T.Sasao \\ P.Wilson
}

\section{INTRODUCTION}

In the term 1990.5-1993.5 the work of the IAU Commission 19 encompasses a broad range of activities with:

- the International Earth Rotation Service (IERS) (director of the Central Bureau: M.Feissel)

- the Commission 19 Working Group on Earth Rotation in Hipparcos Reference Frame (chairman: J. Vondrak) two intercommission working groups :

- on Astronomical Standards (chairman: T. Fukushima)

- on Reference Frames (chairman: Ch.de Vegt)

- the IAG/IAU Special Study Group on Rapid Earth Orientation Variations (chairman: J.O.Dickey)

and independent institutions as well as individual members of the IAU Commission 19. Their reports are shortly reviewed. Investigations of Earth rotation, their correlations with variations of atmospheric angular momentum (AAM) and of terrestrial and celestial reference frames have been developed strongly. In addition to the development of Satellite and Lunar Laser Ranging (SLR and LLR), Very Long Base Interferometry (VLBI), the new observational technique, Global Positioning System (GPS) was introduced to routine determinations of the Earth rotation parameters(ERP) with a high accuracy of the milliarcsecond level and a high resolution of hours. Two international GPS observational campaigns GIG'91 and IGS' 92 in conjunction with the SEARCH'92 were organized in 1991 and 1992 respectively. Diurnal and semidiurnal oscillations of the ERP were determined from GPS and VLBI observations, and also from SLR data and well explained by tidal effects. The influence of atmosphere, ocean and undergroundwater on high frequency variations of Earth rotation has been widely investigated and better understood. The appropriate theoretical studies were carried out in order to improve formula needed for determinations of the EOP (Earth Orientation Parameters) in a case of such highly precise observations as well as for geophysical interpretations of determined variations of Earth rotation. The nutation theory for the rigid Earth was improved and nutation observational models were worked out. In this period there were many international scientific conferences at which the new achievements in this field were presented:

1. Annual IERS Workshops held in Paris in 1991, in Mizusawa in 1992 and in Paris in 1993.

2. IAU Colloquium No 127th on Reference Systems held in Virginia Beach, VA, USA in October 1990.

3. XX IUGG General Assembly held in Vienna in August 1991 and its Symposia.

4. Chapman Conference on Geodetic VLBI: Monitoring Global Change held in Washington, DC, in April 1991.

5. The third Satellite Positioning Symposium held in Columbus, Ohio, USA, in March 1992.

6. IAU Symposium No 156 on Developments. in Astrometry and Their Impact on Astrophysics and Geodynamics, held in Shanghai, China, in September 1992.

7. IAG Symposium No 112, 7th International Symposium Geodesy and Physics of the Earth, Potsdam, Germany,held in October 1992.

8. Annual Meetings of the European Geophysical Society in 1991, 1992, 1993 held in Wiesbaden, Germany and Edinburgh, Scotland.

9. Third Orlov's Conference on Investigations of the Earth as a Planet by Astronomical, Geodetical and Geophysical Methods, held in Odessa, Ukraine, in September 1992.

10. International Workshop for Reference Frame Establishment and Technical Development in Space Geodesy (IRIS'93) held in Tokyo, in January 1993.

11. The 1993 GPS Workshop, held in Bern, Switzerland, in March, 1993.

12. Spring and Autumn Meetings of the American Geophysical Union in 1990-1993, USA.

13. World Space Congress 92, The Orientation of the Planet Earth as Observed by Modern Space Techniques, held in Washington, DC,USA, 1992.

About 200 papers concerning Earth rotation as well as terrestrial and celestial reference frames are indicated by the Astronomy and Astrophysics Abstracts, Vol. 51-55, from the considered period of time. 


\section{REPORT OF THE INTERNATIONAL EARTH ROTATION SERVICE (IERS)}

\section{INTRODUCTION}

The IERS, a member of the Federation of Astronomical and Geophysical Services (FAGS) was created in 1988 by the International Union of Geodesy and Geophysics (IUGG) and the International Astronomical Union (IAU). It maintains: a terrestrial reference frame, a celestial reference frame, and it monitors the Earth Orientation Parameters (EOP), which relate the two frames as a function of time. The IERS plays a key role in the coordination of the observations and of their analysis, setting up and making available the indispensable basic references to the scientific community. Nowadays, the global observing activities involves VLBI, LLR, GPS and SLR with worldwide networks totaling 150 sites in 1992 . The analyses performed by over 30 groups in 10 countries provide the daily monitoring of the Earth's rotation irregularities, the determinations of terrestrial reference frames, crustal motions, as well as the unification of celestial reference frames used in space geodesy and in space navigation with the directions of the extragalactic quasars. Most of the results are accurate and precise at the level of $0.0003^{*}$, corresponding to $1 \mathrm{~cm}$ on the surface of the Earth. While these high accuracy results are used every day in space research programs such as orbit maintenance, space oceanography, space navigation, they also provide unique material for the study of the Earth's interior, crustal deformations, or global dynamics of the oceans and atmosphere, with time characteristics ranging from hours to centuries.

The IERS consists of Directing Board (DB), Central Bureau (CB) with three sections of Terrestrial Frame, Celestial Frame and Earth Orientation, two sub-bureaus(SB) for Rapid Service and Predictions (NEOS) and Atmospheric Angular Momentum (SBAAM), Coordinating Centers (CC) for each of the present observing techniques: VLBI, LLR, SLR and GPS. The IERS is supported by many organizations that contribute to the tasks of observations and data processing. There are about 90 corresponding members of the IERS. The IERS structure and responsibilities were described in details in the previous volume of the IAU Transaction Vol. XXIA, 169-178pp and in the IERS Annual Reports. In 1992 the chairman of the DB was changed on the proposition of Dr. K. Yokoyama, the chairman of the DB in the term 1988-1992. Dr.Y. Yatskiv was elected for the next triennial by the DB. The DB met in the last term five times. The IERS Workshops were held each year: at the Observatoire de Paris in 1991 and 1993 and at the National Astronomical Observatory in Mizusawa in 1992. At the IERS Workshop the reports of the IERS activities and the recent achievements of determinations and investigations of Earth rotation and nutation variations were presented and future programs were discussed.

The IERS published regularly weekly Bulletin A, Monthly Bulletin B, Annual Report, Special Bulletin C,D and Technical Notes. The following IERS Technical Notes were published in the last triennial period:

No 8 - Earth orientation and reference frame determinations, atmospheric excitation functions, up to 1990, (Annex to the IERS Annual Report for 1990)",IERS Analysis Centres for VLBI,LLR,SLR,AAM

No 9 - ITRF 90 and other realizations of the IERS Terrestrial Reference System for 1990, C.Boucher, Z.Altamimi No10 - The IERS GPS Terrestrial Reference Frame, C.Boucher, Z.Altamimi

No11 - Earth orientation, reference frames and atmospheric excitation functions submitted for the 1991 IERS Annual Report, VLBI, LLR, GPS, SLR and AAM IERS Analysis Centres, P.Charlot (ed.)

No12 - ITRF 91 and its associated velocity field, C.Boucher, Z.Altamimi, L.Duhem

No13 - IERS Standards (1992), D.D.McCarthy (ed.)

The mailing list of the IERS publications includes 600 addresses in 65 countries. The main results are also available through electronic means.

\section{THE IERS CENTRAL BUREAU ACTIVTTY}

The IERS Standards (D.D.McCarthy -NEOS, Washington, D.C. USA)

The IERS reference System is composed of the IERS Standards, the IERS terrestrial and celestial reference frames and the corresponding series of the Earth Orientation Parameters (EOP) which are consistent with one another at the milliarcsecond level.

The IERS Standards consist of a set of constants and models used by the IERS. The new revised edition of the IERS Standards (1992) was completed by D.D.McCarthy and published in the IERS Technical Note No13. It replaced the IERS Standards published in 1989 and contains 14 chapters covering the following topics:

1. Numerical Standards, 2. Celestial Reference System, 3. Conventional Terrestrial Reference Frame, 4. Lunar and Planetary Ephemerides, 5. Transformation between the Celestial and Terrestrial Systems, 6. Geopotential, 7. Solid Earth Tides, 8. Ocean Tide Model, 9. Local Site Displacement, 10. Tidal Variations in the Earth's Rotation, 11. Tropospheric 
Model, 12. Radiation Pressure Reflectance Model, 13. General Relativistic Models for Time, Coordinates and Equations of Motion, 14. General Relativistic Models for Propagation.

\author{
The IERS Terrestrial Reference Frame \\ (C.Boucher - CB Terrestrial Frame Section, Institut Geographique National, Paris, France)
}

The IERS terrestrial system is made geocentric through the use of LLR, GPS and SLR observation techniques. Its realization also includes VLBI results, which enables the tie of the terrestrial reference frame to the extragalactic reference frame. A key aspect of this combination is the collocation of observing stations of the various techniques operated in common sites, either in a permanent mode or by repeated occupations. A large proportion of the involved observing sites participate in long range Earth orientation monitoring programs, which strengthens the control of the long term deformations of the Earth's crust. The IERS Terrestrial Reference Frame (ITRF) includes of about 150 site positions derived from the four observing techniques, and site velocities derived from SLR and VLBI data. The velocity field has no rotation relative to the geological plate motion model NNR1-NUVEL. After the ITRF0, the initial IERS-ITRF, new improved systems were worked out each year: the ITRF90, 91 and , 92. They are described in detail in the IERS Technical Notes.The ITRF92 contains in total 281 stations located in 152 sites spread over 8 tectonic plates. Their position and velocity accuracies are of the order of $1-3 \mathrm{~cm}$ and $1.5 \mathrm{~mm} / \mathrm{year}$ respectively.

The IERS Celestial Reference Frame (J.F.Lestrade - CB Celestial Frame Section, Observatoire de Paris, France)

The IERS Celestial Reference Frame (ICRF) is based on the directions of compact extragalactic radio sources observed with VLBI by several national and international programs. It is extended and improved as new information becomes available, while maintaining the stability of its reference directions within 0.1 milliarcsecond. The ICRF90,91,92 were worked out. The origin of the reference system is at the barycentre of the Solar System. Its polar axis and origin of right ascensions are consistent with the FK5 System at J2000.0, and the accuracy of their realization is better than 0.01 ", a two orders of magnitude improvement relative to the ground-based stellar realizations. Its tie with the dynamical frame used in space navigation is known within a few milliarcseconds, thanks to the participation of Lunar Laser Ranging together with VLBI in the Earth rotation program. Its tie with the galactic frame established on the Hipparcos mission as well as the time evolution of the tie will be known within the uncertainty of the Hipparcos frame itself. The core of the most regular observed objects in the ICRF, included in VLBI Earth rotation monitoring programs, insures an excellent tie of the satellite geodesy systems, through the unification of terrestrial frames and the Earth rotation determinations. The characteristics of the ICRF are summarized in Table 1.

Table 1. The IERS Celestial Reference Frame, ICRF91: 422 objects.

$1 a$ - The extragalactic frame, observing techniques: VLBI. Stability of the reference axes: $\pm 0.0001^{\text {" }}$

\begin{tabular}{|l|c|c|c|c|c|c|c||}
\hline Type of object & $\%$ & $\begin{array}{c}\text { Total flux } \\
\text { (Jansky) }\end{array}$ & $\%$ & Redshift & $\%$ & $\begin{array}{c}\text { Precision } \\
\left(0.0011^{\prime \prime}\right.\end{array}$ & $\%$ \\
\hline Quasars & 68 & $0-1$ & 29 & $0-1$ & 47 & $<0.5$ & 61 \\
\hline Galaxies & 6 & $1-2$ & 45 & $1-2$ & 36 & $0.5<<1.0$ & 21 \\
\hline B1Lac & 12 & $2-4$ & 18 & $2-3$ & 13 & $1.0<<2.0$ & 9 \\
\hline Other & 14 & $>4$ & 8 & $3-4$ & 4 & $>2.0$ & 9 \\
\hline
\end{tabular}

$1 b$ - Tie with other celestial frames

\begin{tabular}{|c|c|c|c|}
\hline Scale & Observing techniques & Objects & Precision of the tie \\
\hline $\begin{array}{c}\text { Solar System } \\
\text { Galaxy }\end{array}$ & $\begin{array}{c}\text { Lunar Laser Ranging } \\
\text { Hipparcos }\end{array}$ & $\begin{array}{c}\text { Earth, Moon } \\
11 \text { radio stars }\end{array}$ & $\begin{array}{c} \pm .005^{\mathrm{n}} \\
\pm 0.001^{\mathrm{N}} ; \pm 0.0005^{\mathrm{n}} / \mathrm{y}\end{array}$ \\
\hline
\end{tabular}


The IERS Earth Orientation Parameters (M.Feissel - CB, Earth Orientation Section, Observatoire de Paris, France)

The spectrum of the Earth's rotation irregularities is extremely broad, from hour to centuries, reflecting the free and forced oscillations of the various layers of the planet. Most of the irregularities are aperiodic and poorly predictable, hence the need of monitoring their variations. At present they are represented by five Earth Orientation Parameters (EOP), four to describe the motion of the rotation axis relative to the Earth crust $(x, y)$ and the inertial frame described by the present theories of the precession and nutation ( $d \psi, d \varepsilon$ ), and one (UT1) to describe the angular rotation of the Earth. The EOP series are determined by the IERS Analyzing Centers yearly with taking into account new observations. Detail information about these EOP series are published in the IERS Annual Reports. The main features of the IERS series of EOP are summarized in Table 2.

In 1993 the following number of EOP series were determined by the IERS Analyzing Centers:

VLBI: 14 series (GIUB, SFC, JPL, NOAA, OPA, SHA, USNO)

LLR : 3 series (JPL, SHA, UTXMO)

SLR : 13 series (CLG, CSR, DUT, GAOUA, GFZ, GSFC)

GPS : 6 series (CODE, CSR, EMR, ESOC, JPL, SIO)

COMBINE : 3 series (JPL, NEOS, IERS)

Glossary:

CODE Centre for Orbit Determination in Europe, Switzerland

CSR Center for Space Research, University of Texas, USA

DUT Delft Univ. of Techn., The Netherlands

EMR Energy, Mines and Resources, Canada

ESOC European Space Operation Centre

GAOUA Main Astron.Obs.of Ukraine Acad.of Sciences, Ukraine
GFZ GeoForschung Zenter, Potsdam, Germany

GIUB Geodetic Institute of the University of Bonn, Germany

GSFC Goddard Space Flight Center, MA, USA

IERS International Earth Rotation Service, Paris, France

JPL Jet Propulsion Laboratory, CA, USA

NEOS National Earth Orientation Service, Washington DC, USA

NOAA National Oceanic and Atmospheric Administration, MA ,USA

OPA Observatoire de Paris, France

SHA Shanghai Observatory, China

SIO Scrips Institution of Oceanography, CA, USA

USNO U.S. Naval Observatory, Washington, USA

UTXMO Dept. of Astronomy, Univ. of Texas, USA

Table 2. The IERS Earth Orientation Parameters.

\begin{tabular}{|c|c|c|c|c|c|c|c|c|c|}
\hline \multirow{2}{*}{$\begin{array}{c}\begin{array}{c}\text { Period } \\
\text { (years) }\end{array} \\
1900-1961 \\
\end{array}$} & \multirow{2}{*}{$\begin{array}{c}\begin{array}{c}\text { PM, } \\
\text { UT,CP }\end{array} \\
\text { PM } \\
\end{array}$} & \multicolumn{6}{|c|}{ Observing technique contribution } & $\begin{array}{c}\text { Times } \\
\text { resolution }\end{array}$ & Precision \\
\hline & & $*$ & & & & & & month & $0.05^{n}$ \\
\hline $1962-1972$ & $\begin{array}{l}\text { PM } \\
\text { UT }\end{array}$ & $\begin{array}{l}* * \\
* *\end{array}$ & & & & & & weeks & $\begin{array}{l}0.02^{n} \\
0.002 \mathrm{~s}\end{array}$ \\
\hline 1972-1979 & $\begin{array}{l}\text { PM } \\
\text { UT }\end{array}$ & $* *$ & ** & ** & & & & week & $\begin{array}{l}0.01^{\prime \prime} \\
0.001 \mathrm{~s}\end{array}$ \\
\hline $1980-1983$ & $\begin{array}{l}\text { PM } \\
\text { UT }\end{array}$ & & $*$ & & $\begin{array}{l}* * * \\
* *\end{array}$ & * & & week & $\begin{array}{l}0.002^{\prime \prime} \\
0.0004 \mathrm{~s}\end{array}$ \\
\hline $1984-1991$ & $\begin{array}{c}\text { PM } \\
\text { CP } \\
\text { UT }\end{array}$ & & & & $\begin{array}{l}* * \\
* \\
\end{array}$ & $\begin{array}{l}* * \\
* * * * * \\
* * *\end{array}$ & & days & $\begin{array}{l}0.0005^{n} \\
0.0005^{n} \\
0.00005 \mathrm{~s}\end{array}$ \\
\hline 1992 & $\begin{array}{l}\text { PM } \\
\text { CP } \\
\text { UT }\end{array}$ & & & & $\begin{array}{l}* * \\
*\end{array}$ & $\begin{array}{l}* * \\
* * * * * \\
* * * *\end{array}$ & $\begin{array}{l}* * \\
*\end{array}$ & day & $\begin{array}{l}0.0002^{\prime \prime} \\
0.0005^{n} \\
0.00005 \mathrm{~s}\end{array}$ \\
\hline
\end{tabular}

Notes. The number of * roughly reflect the level of contribution(precision and density of measurements). PM: polar motion $(x, y)$; UT: universal time; CP: celestial pole coordinates(d $\psi \sin \epsilon, d \epsilon)$ 


\title{
THE IERS SUBBUREAUS ACTIVITY
}

The IERS Sub-bureau for Rapid Service and Predictions (D.D.McCarthy - NEOS, Washington, D.C. USA)

The U.S. Naval Observatory (USNO), as a partner in the National Earth Orientation Service (NEOS) with the National Oceanic and Atmospheric Administration (NOAA), continued to function as the Sub-bureau for Rapid Service and Prediction of the International Earth Rotation Service (IERS) publishing IERS Bulletin A weekly, NEOS Annual Report and distributing information by mail, electronic mail and computer bulletin board. The prediction of the correction to the current nutation theory was modified to account for the most recent observations. Predictions of polar motion, UT1-UTC, nutation were provided routinely. Weekly analysis of Earth orientation data derived from GPS orbits was begun by D.D. McCarthy. The information is provided to the International GPS Service (IGS) for distribution to its users. The GPS data were found to make a valuable contribution to the combination with other contributed data, and to improve the rapid service estimates and the predicted Earth orientation parameters. B.J. Luzum designed software to combine the different GPS Earth orientation series to provide a single GPS combination series. A special prediction file to be used by all participating in the IGS is now being produced routinely. The study of the application of atmospheric angular momentum data to the estimation of Earth orientation was continued. Corrections to the main zonal tide components in the variation of the length of day were derived in the process and used to derived corrections to the zonal tidal coefficients for UT1-UTC.

The IERS Sub-bureau for Atmospheric Angular Momentum of the IERS

(Reported by D.A.Salstein, Atmospheric and Environmental Research Inc.(AER),Cambridge, Mass.USA; D. Kann, Research and Data Systems Corp. Greenbelt,M.D.USA)

Since its start date of 1 October 1989, the operations of the Sub-bureau for Atmospheric Angular Momentum (SBAAM) have steadily come to maturity with regular inclusion of atmospheric excitation function from four meteorological data centers. Such functions relate directly to excitations of variations in length of day and polar motion of the Earth. The SBAAM, housed at the Climate Analysis Center of the U.S.National Meteorological Center (NMC) in Washington,D.C., calculates parameters based on wind and surface pressure analyses and forecasts from the NMC global model. Additionally, similar values are received via rapid transmission from the United Kingdom Meteorological Office, the European Centre for Medium-Range Weather Forecasts, and the Japan Meteorological Agency. The parameters are currently produced as frequently as four time per day. Data back to 1976 are available as well, at daily or twice-daily resolution. Studies based on geodetic and SBAAM atmospheric data have addressed agreement between variations in the momentum of solid Earth and atmosphere occurring on a range of time scale from interannual to interseasonal, with tvidence for significant coherence between the two as short as $\mathbf{8}$ days. The Sub-bureau has studied the differences amongst the function produced by the various meteorological centers, which tend to agree down to even shorter time scales. Forecasts of angular momentum quantities out to ten days are also produced by the SBAAM and their skill has been estimated to be positive during these lead time.Such atmospheric data have already been used successfully in forecasts of length of day. Additional quantities produced by the SBAAM include values of zonal mean winds, which are a regional basis for the global axial angular momentum parameter, as well as zonal mean temperature fields. Also the impact of the global ocean is included through computations of pressure-based excitation values modified by the so-called inverted harometer (IB) approximation. Using the IB modification, the distribution of ocean mass responds to atmospheric pressure forcing, with the pure IB response implying a drop in local sea level of $1 \mathrm{~cm}$ per millibar rise in overlying pressure. Other parameters produced by the SBAAM include global mean surface pressure, and a number of low-order harmonics of surface pressure, useful to both geophysical studies and those of satellite orbit determination.

Angular momentum data are distributed to an international group of scientists for atmospheric and geodetic studies both directly from the Sub-bureau as well as from archives maintained at NMC and AER. Further information can be found in Salstein et al., 1993, Bulletin of the American Meteorological Society, 74, 67-80.

\section{THE IERS COORDINATING CENTERS ACTIVITY}

\author{
Satellite Laser Ranging - SLR (B.E.Schutz, Center for Space Research (CSR), Austin, Texas, USA)
}

In October 1992, Lageos-II was launched, an important addition to the existing set of SLR satellites used for Earth rotation and reference frame studies, namely, Lageos-I, Etalon-I and Etalon-II. The performance of the global network of SLR stations has been good, with about the same number of stations (32) contributing about 2100 passes on both Lageos-I and Lageos-II in the same period. Lageos-II has an inclination of 53 degrees compared to 110 degrees for Lageos-I and both satellite have an altitude of about $5900 \mathrm{~km}$. Tracking of the Etalon satellites has, unfortunately, not yet reached a level that would make these satellites regular contributors to Earth rotation. On the average, the Etalon tracking 
was slightly more than one pass per day. During 1992, scheduling modifications were recommended to allow partitioning of an Etalon pass into periods when other satellites can be tracked. The full implementation of these considerations has not been made at all stations. SLR data from the past decade have been a significant contributor to new gravity fields, for example, JGM-1 and JGM-2 prepared as a joint activity of the NASA Goddard Space Flight Center and the University of Texas at Austin. Data from Lageos-II is expected to further "tune" the gravity field and produce a new field for Earth rotation and reference frame applications.

Very Long Base Interferometry - VLBI (W.E.Carter - National Geodetic Survey -NGS; Gravity, Astronomy and Satellite Branch, USA Dept.of Commerce, Rockville MD,USA)

Since mid-1990 the number of VLBI observations has increased significantly, including in the southern hemisphere where VLBI facilities had been very sparse. In chronological order: the Italian Space Agency (ASI) built a new geodetic VLBI facility at Matera, Italy, which began operations in 1990; a Japan facility at Syowa, Antarctica, made its first observations in 1990; a format NASA tracking antenna at Santiago, Chile, was converted to geodetic VLBI use in 1991, through the joint efforts of Institute of Applied Geodesy (IFAG), Germany, the National Aeronautics and Space Administration (NASA) and the National Oceanic and Atmospheric Administration (NOAA), United States, and Center for Space Studies, University of Chile; IFAG established a new observatory at the Chilean Antarctic station O'Higgins during 1992; the Canada Center for Surveying upgraded the Algonquin Park station and began participating regularly in international Mark III observing sessions in 1992; and NOAA, the University of Sao Paulo, and the Brazilian Space Agency (INPE) made the first successful observations from a jointly operated new facility near Fortaleza, Brazil, in April, 1993. Work is continuing on the Russian (former USSR) Quasar network and joint Institute for Applied Astronomy, Russia and NASA experiments are planned for mid 1993. A new observatory is also under construction at Ny Alesund, Spitzbergen, by Statens Kartverk, Norwegian Mapping Authority. In the United States, the Mojave station was closed in July 1992, and the Richmond station was destroyed by Hurricane Andrew in August 1992. However, the U.S. Naval Observatory has nearly completed a new $20 \mathrm{~m}$ telescope at Kokee Park, Hawaii, and similar telescopes will be built at Green Bank, West Virginia, and Richmond, Florida, within the next year. The 10-station Very Long Baseline Array (VLBA) network will become fully operational during 1993 with observatories spanning North America, from the Virgin Islands to Hawaii. When fully operational these new facilities will provide a global network of reasonably well distributed VLBI stations to define and maintain both the terrestrial and celestial reference frames. The new observations in the southern hemisphere have already been especially significant in expanding the celestial reference frame. Between 1990 and 1992, the IERS Celestial Reference Frame grew from 228 to 422 sources, much more uniformly distributed about the sky. The coordinates of 81 sources observed in the IERS programs, ranging in declination from 78 degrees north to 80 degrees south, have formal errors of a few tenths of a millisecond of arc (Robertson et al., 1993; A.J., 105,1,3353-3358).

Haystack Observatory is developing a next generation Mark IV VLBI data acquisition system, with joint support from NASA, NOAA, USNO and IFAG. The Mark IV system will have double the sensitivity of the Mark IIIA system by increasing the maximum data rate to $1024 \mathrm{Mbits} / \mathrm{sec}$. In addition, the Japan Communications Research Laboratory is developing an upgraded K4 and the Canadian Institute for Space and Terrestrial Science is developing the highly transportable S2 system, both of which use rotary-head cassette recorder systems.

Two large meetings with major VLBI emphasis have been held since mid-1990. The "Chapman Conference on Geodetic VLBI: Monitoring Global Change" was sponsored by NOAA and held in Washington, DC on 22-26 April 1991. The Japanese Science and Technology Agency held the "International Workshop for Reference Frame Establishment and Technical Development in Space Geodesy" (IRIS'93) in Tokyo on 18-21 January 1993. Proceedings volumes bave been published for both meetings.

Dong and Herring (1990; EOS,71,482) reported unambiguous detection of EOP variations in the diurnal and semidiurnal bands due to oceanic tidal effects. The variations had been predicted a decade earlier by Yoder et al.(1981; JGR,86,881-891). Subsequent VLBI, GPS and SLR analyses have supported the Dong and Herring results and routine EOP analyses have begun to take these effects into account.

Global Positioning System - GPS (W.G.Melbourne - Jet Propulsion Laboratory, Pasadena, CA, USA)

During the past decade over two orders of magnitude in accuracy has been achieved in the application of GPS to Space Geodesy. The period 1990-1993 has been a time of expanded utility of GPS geodetic systems to a broader spectrum of scientific applications, not only because of their potential for high accuracy, but also for their technical and economic capability to sharpen spatial and time resolutions and to complement established space geodetic techniques. The figure shows baseline repeat abilities reported over the last decade by a number of investigators for a wide range of baseline lengths.

In 1990 the IERS Directing Board set up an interim Technique Coordinator for GPS to evaluate its potential for EOP applications and to encourage broader participation in the use of the technique.The GIG'91 campaign was a first 
attempt of coordinating, on a international basis, the efforts of a number of participants in developing a globally distributed network of GPS tracking stations. The campaign was executed over a three-week period in Jan-Feb 1991. Its primary purpose was to collect tracking data with sufficient strength to evaluate the accuracy of the GPS ephemeris products, the tracking station locations and the EOP information. A subsequent workshop sponsored by "Institute fur Angewandte Geodasie" in Ahrweiler, Germany, in Aug. 1991, verified the expected performance of GPS. There are now a total of eight GPS Analysis Centers contributing on a regular basis their EOP\&TRF results to the IERS Central Bureau. As results of these IERS activities and partially out of recognition of the enormous potential of GPS, if properly coordinated, a small working group was formed in early 1990 with the goal of establishing a permanent GPS service under the IAG auspices for scientific applications (see the paragraph on the International GPS Service for further information ). The IERS participated in the organization of the highly successful summer-long campaign IGS'92 to further evaluate GPS performance but also to evaluate the ability of the participating organizations to support on a permanent basis the requirements of the Service. An additional activity was the SEARCH'92 campaign, which coordinates these GPS activities with those from SLR and VLBI to support short period Earth rotation studies. The 1993 GPS Workshop reviewing results of the GPS'92 Campaign was held at the Technical University in Berne, in March 1993. The Berne meeting also provided the opportunity to establish agreements with the IERS regarding IERS/IGS interface questions and respective areas of responsibility. In effect, the IERS will depend on the IGS for operation of the GPS network and delivery of GPS data products. The IGS will use IERS Standards and depend on the IERS for EOP and Terrestrial Reference Frame information.

\section{REPORTS OF THE WORKING GROUPS}

IAG/IAU Special study group on rapid Earth orientation variations (chairman: J.O.Dickey, JPL, Pasadena, USA)

Special Study Group, Rapid Earth Orientation Variations 5.143, a joint International Association of Geodesy and International Astronomical Union (IAG/IAU) SSG, has been formed to (1) interface with the IERS in the determination of rapid variations in Earth rotation by the space geodetic techniques; (2) advocate for the best possible auxiliary data from geophysical, oceanographic and atmospheric sources; (3) encourage improvements in measurement techniques (including geodetic, atmospheric, oceanographic and geophysical); and (4) encourage cooperative multidisciplinary studies.

A special measurement campaign, SEARCH'92 (Study of Earth Atmosphere Rapid Changes) was held during the Summer of 1992 (June 21 - September 22, 1992) in conjunction with the GPS experiment sponsored by the International GPS Service; the coordination was effected through the IERS. The campaign involved all space techniques and the best available complementary geophysical, oceanographic and atmospheric data were obtained. Spacial efforts were successful in obtaining 6-hourly AAM determinations as well as the routine calculation of atmospheric torques. Plans are now being planned for a complementary experiment to be held in Northern Hemisphere in the winter of 1995 . The scientific benefits to be obtained from these campaigns include increased understanding of the properties and origin of short-period fluctuations in the Earth's orientation, improvements to the tidal model at sub-monthly periods, and improved ability to predict changes in the Earth's rotation up to a month in advance. Several sessions on this topic and of the SSG 5.142 have been held in conjunction with scientific conferences. Minutes of the SSG5.142 meetings were printed and distributed.

\section{IAU Commission 19 Working Group on Earth rotation in Hipparcos Reference Frame} (Chairman: J.Vondrak, Astronomical Institute, Prague, Czech Republic)

The Working Group has continued in preparing the new global solution of Earth Orientation Parameters from optical astronomy since the beginning of the century, referred to the Hipparcos catalog now under preparation (Capitaine,1991; Vondrak, Ron, 1993). Individual star observations will be used, instead of group means used in the past, and a number of additional parameters will be estimated from a single adjustment. The main effort was on two directions: 
i) Collection of the observations from the observatories and their preliminary analysis; ii) Preparation of algorithms and softwares to be used and their tests.

The final list of observatories selected to participate comprises 55 different instruments working at 37 observatories in 19 states. At present, about 50\% of data is collected at the Astronomical Institute in Prague and the data flow proceeds well. The data files are analyzed for possible systematic effects and reformatted into standard formats, according to the types of instruments. The search for systematic effects allow to detect the systematic deformations of the almucantar of the order of $0.1^{\prime \prime}$ in Paris and Chile and significant color and magnitude effects of the same order for astrolabe anf transit instruments which have to be adjusted as a new parameters in the global solution ( Hefty, 1991; Bougeard, 1992, 1993; Peek et al., 1993). The problems of refraction have been studied in Poland (Kurzynska, 1991) in order to improve the models of refraction originally used at the observatories to reduce their data in the past and to recalculate the observations in a unique manner. A relatively simple refraction formula was derived on the basis of using both the Laplace formula and a numerical integration. To use it, meteorological data changes during each night are needed (temperature, air pressure and also air humidity).

Statistical studies have been made in France (Bougard et al., 1991) in order improve modeling, detection and separation of perturbing effects, using the most recent findings of mathematical statistics. The main effort was concentrated on three topics:

a) multivariate statistical procedures to detect and separate sources of perturbing effects in optical observations,

b) a posteriori (linear or nonlinear) modeling of magnitude color and sidereal time effects in the reduction model,

c) elaboration of numerical algorithms for robust fitting on large data sets.

The problem of simultaneous determination of polar motion and UT1-UTC with celestial pole offset was studied. It was found that the use of celestial pole offset has certain advantage over classic $z$ and $w$ terms used in the past (Vondrak et al., 1993). The time evolution of the accuracy expected from the prepared solution was estimated from the known precision and distribution of the observations in space and time. Polar motion of a 5-day solution can be known to $\pm 0.030^{\prime \prime}$ in the first half of the century and $\pm 0.009^{\prime \prime}$ after 1960 , universal time can be determined to $\pm 0.0008 \mathrm{~s}$ after 1960. The expected accuracy of the celestial pole offset is of the order $\pm 0.02^{n}- \pm 0.08^{n}$ at the beginning of the century and $\pm 0.01^{\prime \prime}$ during the last two decades.

The prepared software has been tested first with simulated data and then with the ILS observations between 1900 and 1978, using the orginal star catalog. The results proved that the proposed algorithms are capable of providing new interesting information on EOP (Vondrak, 1993).

The WG met three times at the period covered by this report; at the "Journees 1991 Systemes de Refereace Spatio-Temporels - Geodynamique Globale and Systemes de Reference" (Paris, June 1991), at the IAU Symposium N.156 "Developments in astrometry and their impacts on astrophysics and geodynamics"(Shanghai, September 1992) and during the VIII Lohrmann Colloquium "Geodetic Astrometry"(Dresden, March 1993).

IAU Intercommission Working Group on Astronomical Standards

(Chairman: T. Fukushima, Hydrographic Department of Japan, Tokyo, Japan)

Since its establishment in 1991, the IAU WG on Astronomical Standards has worked actively through e-mail based correspondences and by issuing 3 questionnaires on the given themes: the way of electronic distribution, the collection of standard softwares and constants, and answering some questions on time. The responses seem to support the idea of establishing an international center plus a board of reviewing for the maintenance of "IAU Standards", which will be liked to the astronomical version of the IERS Standards. The differences between the IAU and IERS Standards are not only in their scopes. The IAU Standards will be distributed in a machine-readable form. The test collection of standard softwares was held in the first half of 1993 with a focus on the routines computing the precession in FORTRAN. The diversity of interfaces of submitted routines has raised a serious question on the policy of creating IAU Standards; whether we adopt a standard interface or not.

IAU Intercommission Working Group on Reference Frames (Chairman: Chr. de Vegt, Hamburg Observatory, Germany)

Working Group on Reference Frames focus its work on preparation of a revised list of candidate extragalactic sources as well as a list of radio stars and stars for use with the new optical astrometric interferometer (USNO+NRL). They will be discussed in March 1994 and a preliminary report will be available at the IAU General Assembly 1994. 


\section{THE INTERNATIONAL GPS GEODYNAMICS SERVICE (IGS)}

In March 1990, at the IAG Executive Committee meeting in Paris the IAG Planning Committee for the IGS was formally established. The call for participation was issued 1 February 1991 . More than 100 proposals made it possible to structure the observation network (30 core stations equipped with precise P-Code receivers and efficient data links), and to organize the data flow (data centers on different levels) and processing. At the XX-th General Assembly of the IUGG in Vienna, in August 1991, the Planning Committee was reorganized and named the IGS Campaign Oversight Committee; The IUGG resolution No 5, recommending that the concept of the IGS be explored over the next four years, was adopted. More information about the early phase of IGS may be found in the papers: (Mueller, 1992), (Mueller and Beutler, 1992), (Beutler, 1992).

The 1992 IGS Test Campaign (21 June - 23 September) and the intensive observation campaign called Epoch'92 were organized by the IGS Campaign Oversight Committee. The declared goal of the three-month 1992 IGS Test Campaign was the routine production of accurate GPS orbits and determinations of the rapid Earth rotation. IGS Epoch Campaigns will be organized about every second year. The primary goal is a first densification of the sparse IGS Core Network. At the 3rd IGS Oversight Committee meeting, in October 1992 at Goddard Space Flight Center, it was decided to establish the IGS, PILOT SERVICE to bridge the gap between the very successful 1992 IGS Campaign and the start of the routine IGS service. The routine service should start on 1 January 1994. The primary goal of the International GPS Geodynamics Service (IGS) is to give the scientific community high quality GPS orbits on the regular basis and related information like Earth rotation parameters, and to perform regional or local GPS analyses without further orbit improvement.

The 1993 GPS Workshop reviewing results of the GPS'92 Campaign was held at the Berne Technical University in March 1993 (Beutler, Brockman, 1993). At this meeting the agreement with the IERS regarding IERS/IGS interface questions and responsibilities was established.

\section{REPORTS OF INSTITUTE *}

This section summaries the reports received from institutes and IAU Commission members. The Jet Propulsion Laboratory, the U.S.Naval Observatory, the National Astronomical Observatory in Mizusawa, the Shanghai Astronomical Observatory have carried on wide investigations of Earth rotation and are reviewed first.

Jet Propulsion Laboratory, Pasadena, CA, USA (reported by J.O.Dickey). JPL has been actively involved in many areas which briefly can be outlined as follows:

0 the determination of constants and the development of models and ephemerides for use by the community and the IERS; o reference frame studies: 1) establishment of the JPL Radio Frame; 2) the establishment of the Dynamical Reference Frame of the Lunar/Planetary Ephemerides; 3) determination of ties between the various reference systems; 4) development of the concept of the dynamic equinox as a reference point; 5) development of the fiducial-free approach using GPS data and the realization of an independent GPS terrestrial reference frame tied to the IERS.

o acquisition, reduction and analysis of VLBI data, including the TEMPO program, for Earth rotation, precession, and nutation studies;

- reduction and analysis of lunar laser ranging (LLR) data for Earth rotation, precession and nutation studies;

o acquisition, reduction and analysis of GPS data in Earth rotation and reference frame studies;

0 intercomparisons of Earth rotation data of the various techniques and combination of them with a Kalman filter;

0 analysis of the scientific implications of these measurements.

Earth rotation and polar motion studies have clearly demonstrated that an unprecedented accuracies $(0.3$ mas for polar motion and $0.03 \mathrm{~ms}$ for UT1) are now being achieved by the modern space geodetic techniques using high-quality estimates of the atmospheric excitation of Earth rotation and polar motion. It was shown that changes in the (LOD) at seasonal and higher frequencies are dominated by the exchange of angular momentum between the atmosphere and the solid Earth except at tidal periods and are coherent down to 8 days, with lack of coherence at shorter periods caused by the deckling signal-to-measurement noise ratio in both the AAM and LOD data types (Dickey, Marcus, Steppe and Hide, 1992). Using the high resolution GPS measurements along with the non-axial components of the (AAM) chi-functions computed from general circulation models of the atmosphere, it has been shown that atmospheric wind and pressure fluctuations were largely responsible for exciting the rapid polar motions observed during the three-week period of the GIG'91 measurement campaign (Gross, Lindqwister, 1992).

Since 1992, JPL has been routinely producing daily estimates of polar motion with 0.3 mas accuracy using data from a 40-station global network of GPS receivers (Zumberge, Jefferson, Blewitt, Heflin, Webb, 1993).

* List of References indicated in these reports can be obtained on request from the IAU Commission 19. Some of them can be found in the Astronomy and Astrophysics Abstracts. 
An expression was first derived which relates the location of the Celestial Ephemeris Pole (CEP) to that of the rotation pole which is reported by observing programs. The Liouville equation was rewritten in terms of the location of the CEP (Gross, 1992). This newly derived equation can then be used to directly interpret polar motion observations in terms of its geophysical excitation mechanisms (see also A.Brzezinski, at the paragraph of the Space Research Center, Warsaw, Poland).

Subdaily Earth orientation changes have been detected by both the GPS and VLBI observing programs at JPL and have been theoretical interpreted in terms of ocean tidal effects on the Earth's rotation (Lichten, Marcus, Dickey, 1992; Freedman, Ibanez-Meier, Dickey, Lichten, Herring, 1993; Lichten, Marcus, Dickey, 1992; Dickey, Marcus, Lichten, 1992; Sovers, Jacobs, Gross, 1993). The angular momentum carried by the ocean tidal current and sea level height variations can be generated with the angular momentum of the solid Earth, thereby affecting the solid Earth's rotation. The theoretical ocean tidal angular momentum results of Seiler were used to compute the theoretical effect of ocean tides on the Earth's rotation, accounting for the yielding of the solid Earth and the presence of the Free Core Nutation in the diurnal tidal band. These theoretical Earth orientation changes at semi-diurnal and diurnal tidal frequencies agree better with the polar motion observations than they do with the UT1 observations (which can differ with the theoretical predictions by up to a factor of two in amplitude), including the need for further refinements of the ocean tidal models.

JPL has explored the source of the intraseasonal "40-50 day" oscillation by analyzing LOD data in concert with AAM results from both operational analyses and the UCLA Atmospheric General Circulation Model (Dickey, Ghil and Marcus, 1991). Findings indicate that two intraseasonal oscillations exist in the Earth-atmosphere system: a tropical 50-day oscillation, associated with the convectively driven Madden-Julian wave and a mid-latitude 40-day oscillation associated with the interaction of non-zonal flow with topography. On the seasonal time periods, the contributions of the global oceans and in particular that of the Antarctic Circumpolar Current to Earth rotation were investigated (Dickey, Marcus, Hide, Thompson, Johns, 1993).

JPL has linked the El Nino /Southem Oscillation (ENSO) phenomenon to LOD variation. Significant variability of the LOD and Southern Oscillation Index (SOI) is centered at $\sim 4.2$ years (traditional ENSO cycle and a distinct quasi-biennial component (at $\sim 2.3$ years) is observed. During the 1982-1983 ENSO event 90\% of the LOD variance can be explain by the atmosphere. Examining the latitudal redistribution of angular momentum within the atmosphere, we discovered a slow, global-scale poleward AAM propagation that originates in a quatorial regions, where westerly anomales lead the main ENSO anomalies by nearly two years. Coherence sygnals penetrate to latitudes higher than $60^{\circ}$ in both hemispheres, where they lag behind the ENSO cycle by four years (Dickey, Marcus, Hide, 1992). High correlation hetween LOD and SOI is obtained from about 1930 to the present, indicating that LOD can be used as a proxy-index for interannual variations in global wind system after 1930 (Dickey, Marcus, Hide and Eubanks, 1992, 1993).

High-quality observations of the Earth's rotation are being combined using a Kalman filter in order to produce a suite of Earth rotation series of varying duration and temporal resolution (Gross, 1992). Singular Spectrum Analysis (SSA) is being applied to these series in order to separate the meaningful signal from the noise prior to interpreting the signal in terms of geophysical excitation mechanisms such as atmospheric fluctuations or core-mantle interactions. The theoretical effect on the Earth's rotation of the 30 greatest earthquakes to have occurred since 1950 has been computed and found to be large enough to contribute to the observed polar motion variations at detectable levels (Gross, 1992).

The analyses of lunar laser ranging (LLR) data, now over 8,000 normal points from 1969-1993 permit long term studies of variations in the Earth's rotation, as well as determination of many parameters of the Earth-Moon System. The L.LR-determined value of Earth gravity constant is $G M=398600.443 \pm 0.004 \mathrm{~km}^{3} / \mathrm{sec}^{2}$ and of the tidally driven secular acceleration of the Moon is $-25.9 \pm 0.5 \mathrm{arcsec} / \mathrm{cy}^{2}$ corresponding to a linear increase of $3.8 \pm 0.1 \mathrm{~cm} / \mathrm{yr}$ in the mean distance of the Moon. The correction to the IAU-adopted precession constant is $-3.3 \pm 0.4$ milliarcseconds/yr, giving the luni-solar precession constant as $\mathbf{5 0 . 3 8 4 5}$ arcsec/yr at J2000. The amplitude of the 18.6-year nutation of the Earth's pole in space needs to be increased by 3 milliarcseconds (Williams, Newhall, Dickey, 1993). A joint analysis of LLR and VLBI data for precession and nutation corrections utilizes the complementary strength of both techniques (Charlot, Sovers, Williams and Newhall, 1991).

Mizusawa Astrogeodynamics Observatory/NAO, Japan (reported by T. Sasao). Developments of instruments. K-4 VLBI system has been successfully used in many geodetic applications including Earth rotation monitoring (Yoshino, 1991; Kiuchi et al., 1991). A variety of high-performance VLBI terminals and correlators are under development on the basis of the K-4 system (Kawaguchi et al., 1991; Kawaguchi, 1993; Kiuchi et al., 1993; Matsumoto and Kawaguchi, 1993; Tamura et al., 1993). Compatibility of VLBI systems is an increasingly vital problem for the international scientific community (Hama et al., 1993). A new $10 \mathrm{~m}$ VLBI antenna with the phase-stabilized optical-fiber signal transmission system (Sato et al., 1992, 1993) and an advanced digital servo-control system is now in use at Mizusawa Astrogeodynamics Observatory /NAO (Hara et al., 1993; Kameya et al., 1992a, b). The new $10 \mathrm{~m}$ antenna will succeed the role of $6 \mathrm{~m}$ antenna at Nobeyama (Fujishita, 1993) in the international Earth rotation observations. A concept of a new VLBI system with clusters of 4 antennas at each end of a VLBI baseline has been developed for Project VERA (VLBI for the Earth Rotation study and Astrometry). Simultaneous VLBI observations of 4 different radio sources and effective cancellation 
(f) irregular atmospheric refraction effect as well as other sources of systematic errors shall make the new system a promising tool for higher precision and high time-resolution geodesy and 10 microarcsecond level differential astrometry (Kawano et al., 1992; Sasao and Morimoto, 1991; Sasao et al., 1992; Sasao et al., 1993). Experiments for new types of VLBI observations such as Earth rotation monitoring with orthogonal VLBI baselines (Yoshino et a]., 1990), burst mode observations (Yokoyama et al., 1990) and higher frequency observations (Takahashi et al., 1993) have been successfully performed. Effects of atmosphere on VLBI observations were studied in several aspects (Abe et al., 1991; Goto et al., 1991; Manabe et al., 1991a; Sasao et al., 1993). A new SLR station has started observation at Communications Research Laboratory, Koganei, Tokyo since 1990 and successfully obtained many passes for major geodetic satellites (Kunimori, et al., 1993). The station will serve as a collocation point with VLBI. Radio transmitters which are planned to be installed on the surface of the Moon for differential VLBI observations of lunar librations and tidal deformations have been developed and proven to survive after strong impacts with $10000 \mathrm{G}$ decelerations (Hanada et al., 1992; Kawano et al., 1993). Measurable tidal deformations of the lunar surface were estimated in relation to the internal structure of the Moon (Ooe and Hanada, 1992).IERS VLBI Technical Development Center was established in 1990 in Communications Research Laboratory, promoting and coordinating the efforts for developing new technologies in space geodesy (Yoshino, 1993).

Participation in International Earth Rotation Observations. National Astronomical Observatory and Communications Research Laboratory have jointly conducted IRIS-Pacific VLBI observations with Kashima $34 \mathrm{~m}$ and Nobeyama $6 \mathrm{~m}$ antennas on the monthly basis with occasional burst mode observations (Yokoyama et al., 1990). The Hydrographic Department of Japan has carried out a large number of SLR observations at Simosato, which is active as an IERS site since 1988, and at several sites in Japan with a transportable SLR system HTLRS (Sasaki and Sengoku, 1993). Algorithm and physical models for the VLBI Earth Rotation analysis have been improved taking into account achievements in the relevant fields of science. VLBI data obtained at nearly 60 observation sites over the world since 1984 were reanalyzed with the improved software and the results were reported to the Central IERS Bureau. A software was developed to determine the Earth orientation parameters with arbitrary time interval and applied to an analysis of the short time scale variations of the polar motion and UT (Manabe et al., 1991b; Manabe, 1992; Manabe et al., 1993). SLR ETALON data collected during 5-month period including the IERS ETALON Autumn Intensive Campaign were analyzed to evaluate the scientific potential of ETALON in determining the Earth orientation and other geodynamic parameters (Murata and Tsujii, 1992). A new method was developed for improving the fundamental star catalog on the basis of the astrolabe data and applied to the past Danjon astrolabe data collected at Mizusawa. Precise positions were determined for all the observed stars (Manabe and Sakai, 1990). Recent activities of the IERS and VLBI contributions to IERS reference frames and Earth orientation parameters have been summarized (Yokoyama, 1991; Yokoyama, 1992; Yokoyama 1993). A number of Japanese institutions actively took part in observations and analyses for IGS/Epoch' 92 and other global GPS campaigns in order to confirm the feasibility of GPS observations in determination of the Earth rotation parameters and establishment of the global terrestrial reference frame (Hatanaka et al., 1993; Kato and Nakano, 1993; Murata et al., 1993a, b). Results of precise time comparison with LORAN-C and GPS time signals have been carefully examined and some improvements were achieved in the GPS time transfer with application of an advanced analysis software (Horiai, 1992; Horiai and Tamura, 1993).

Physics of the Earth's Variable Rotation. The seasonal budget of the Earth's axial angular momentum has been studied using IRIS VLBI data and numerical models for global atmospheric motion provided by Japan Meteorological Agency. A new quasi-seven-month oscillation ('QSO') was found in both the LOD (length of day) and the tropospheric zonal wind.It was found that the quasi-biennial oscillation, which has been observed in the LOD and the stratospheric zonal wind, exists also in the tropospheric zonal wind. It is confirmed that the inter-annual variations of the LOD with time scales up to five years are mostly explained by the AAM (atmospheric angular momentum) changes due to the oscillations in the tropospheric and stratospheric zonal wind. LOD residual variations with time scales longer than five years, after removal of the inter-annual AAM contributions, are probably related with the core-mantle coupling process (Naito and Kikuchi, 1990, 1991, 1992a,b). Possible non-linear behavior of the oscillations in the AAM and the Earth's rotation was pointed out (Naito, 1992). UT1 variations due to the long period tides were analyzed and the possible range of the mantle $Q$ was discussed (Tamura, 1990, 1993). Free Core Nutation parameters were estimated on the basis of the superconducting gravimeter data obtained at three sites in Japan. The results show closer agreement with the VLBI results compared with other reported SCG-based parameters (Sato et al., 1992). Tidal evolution of the LOD, the lunar orbit and the obliquity of the Earth over the geological past was numerically calculated on the basis of a variable ocean-continent distribution model (Abe et al., 1992a,b). Variations of the plumb line direction are derived from the results of the astronomical time-and-latitude observations and their possible correlation with El Nino events was pointed out (Abe, 1992).

U.S.Naval Observatory, USA (reported by D.D.McCarthy). PZT Observations. Operation of the last PZT 7 in Washington ceased with the observation of 31 December 1991. The total statistics of all USNO PZT data are as follows: Observing sites: Washington, D.C. (PZT's 1, 3, 7) and Miami (Richmond),FL (PZT's 2,6); Observing period: October 
18, 1915 to December 26, 1991; total number of stars observed: 472; total number of nights observing: 26 971; total number of star observations: 675329 . B. Archinal completed gathering USNO PZT data for distribution to the IAU Working Group on "Earth Rotation in the HIPPARCOS Reference Frame".

VBI. The NEOS VLBI network was created from the merger of the NOAA IRIS and the USNO NAVNET networks. This became effective on 1 May 1990. In the three-year reporting period a total of 157 NAVNET experiments of 24-hour duration have been fully processed creating a data base of 71668 delay observations. The accuracy appears to be 0.6 mas in $x, 0.5$ mas in $y$, and $0.05 \mathrm{~ms}$ in UT1-UTC.

The treatment of NAVNET observations was modified by M.Eubanks to include solving for the UT1 rate. Under the guidance of M. Eubanks, the new Hawaii telescope for use in the NAVNET observations was completed in 1993. In a study of NAVNET residuals, D. Matsakis found that the residual scatter is a strong function of the wet path length and it is site dependent as well. The average residual was a function of elevation for all baselines involving Kauai. Software was written to plot the average closure phase violation as a function of baseline and source.

In cooperation with personnel from NASA and Haystack Observatory,K. Kingham continues work on the MARK-IV correlator development. The NEOS Directing Board agreed that investigations should take place into using data from NRAO's new VLBA network for Earth orientation information.

Approximately 800000 frequency-baselines were processed by the Washington Mark IHA VLBI correlator between January 1991 and May 1993. In this time period (20 857 hours), the correlator operated for 21347 hours at a load factor of $102 \%$ (greater than $100 \%$ load factor is made possible by splitting the Mark IIIA correlator to process two sessions simultaneously). The Washington correlator facility shipped and received approximately 9500 tapes, equivalent to 16000 miles or 70 tons of tape.

Prediction of Earth rotation. The prediction of the correction to the current nutation theory was modified by D.D.McCarthy taking into account the most recent observations. Predictions of polar motion, UT1-UTC, and nutation were provided to the IERS (McCarthy, and Luzum, 1991, 1992; McCarthy, 1993).

Reference systems. Work by D.D. McCarthy, M. S. Carter, B. Archinal and D. Florkowski on the construction of an extragalactic reference frame was continued in cooperation with the Naval Research Laboratory (NRL), Hamburg Observatory, and the National Aeronautics and Space Administration (NASA). D.D.McCarthy completed and distributed the second edition of the IERS Standards and B.Archinal wrote the chapter on Terrestrial Coordinates and the Rotation of the Earth for the Explanatory Supplement to the Astronomical Almanac.

Analyses of Earth orientation observations. D.D. McCarthy and B.J. Luzum (1991, 1992) derived corrections to the main zonal tide components in the variation of the LOD and used them to derive corrections to the zonal tidal coefficients for UT1-UTC. D.D. McCarthy investigated various ocean tide models by comparing the predicted variations in LOD with observations. Improved model coefficients nutation were determined by D.D. McCarthy and B.J. Luzum (1992) from observations of nutation. D.D. McCarthy and B.J. Luzum (1991) also completed work on extending the NEOS combination EOP time series back to 1979. B. Archinal continued his investigations on the use of the GEODYN/SOLVE software, with which it could be possible to do direct combination SLR/VLBI solutions. Recent software advances show great promise for allowing combination of SLR, VLBI, and GPS solutions, which in turn should provide much higher accuracy of Earth rotation and reference frames.

Shanghai Astronomical Observatory, Chinese Academy of Sciences, China (reported by Jin Wenjing, Wei Xue, Xu Tongqi). The following investigations were carried out:

- construction of new instruments: $25 \mathrm{~m}$ VLBI antenna with MKIII receiver in Urumqi station, third generation laser with precision of $2-3 \mathrm{~cm}$ in Shanghai and Beijing SLR stations, Urumqi SLR station is under construction;

- Shanghai Observatory is one of the IERS Analysis Centers for VLBI, SLR, LLR data;

- participation in the IGS'92 and SEARCH'92 campaigns with 10 GPS receivers and 3 laser ranging systems;

- collection and reduction of 75293 optical astrometric observations made in 1988.0-1991.0 by 64-42 instruments located all over the world. (Quarterly and Annual Reports on Optical Astronomical Observations, Shanghai 1989-1992). Since 1991 no ERP have been computed from these observations;

- nine Chinese optical astrometric instruments are included in the list of 55 instruments of which ERP results will be rereduced in the Hipparcos reference frame;

- the effects of the systematic difference between FK5 and FK4, as well as the change of astronomical constant systems, on the EOP were studied extensively (Jin et al., 1991; Xu et al., 1991; Li, 1990a; Xu et al., 1990). After the elimination of systematic difference of the catalogs, the accuracy of comparisons between Chinese Joint Universal Time (CJS) and BIH or IERS one are better than old ones ( $\mathrm{Li}, 1990)$;

- effective multistage-multiarc method was introduced into the determination of ERP series and geocentric coordinates by 
processing SLR data;

-new methods like a differential filter, a deconvolution method of Fourier transformation, Marple algorythm of AR spectrum, adoptive AR model have been used for analyses of the pole motion and pole motion predictions;

-UTO data determined by LLR were studied and effects of lunar libration models, relativistic effects, uncertainties ephemeris equinox and the elliptic obliquity were discessed;

-the period and $Q$ value (60-70) of Chandler wobble were derived using Zschau's mantle rheology model, and the effect of the equilibrium pole tide of the inelastic Earth on Chandler wobble was discussed;

- the excitation of atmosphere and a water storage distribution to the polar motion were analyzed showing the correlation of an annual variation of a water storage and of the polar motion;

- short periodical oscillations of the Earth rotation and their relationship with variations of the atmosphere, ocean and solar activities have been studied deeply. Oscillations with periods of 50,90,120 days in LOD and AAM variations were found. Big influence of zonal winds of atmospheric circulation on 50 days LOD oscillation was indicated. The study shows that high frequency oscillations of LOD are caused mainly by medium and low latitude regions of northern and southern hemisphere;

-study of the correlations between solar constant, near-Earth space environment, solar neutrino flux and Earth rotation indicated that the interannual changes of the Earth rotation and El Nino phenomena might be induced by solar activities; -the results of sea level changes (SLC) with Earth rotation were studied on the basis of 30 years of observations of 50 tide gauges located along Pacific and the ERP data from the IERS. Positive correlation was obtained in the eastern Pacific and a negative one in the western Pacific. Dynamical analysis of the angular momentum of the tropical Pacific shows that the tropical Pacific influence account for $30 \%$ of the interannual change of the LOD and more during El Nino events, especially this in 1982-1983. The raise of a sea level in the Pacific with the rate of $0.2 \mathrm{~cm} /$ year in the last 30 years was found;

- the influence of changes of the zonal angular momentum of the Earth's atmosphere in the stratosphere and lower mesosphere on Earth rotation have been estimated with the method of balance of angular momentum. Results show that the solar activity causes the average deviations of the Earth's angular velocity of $1.35 \times 10^{13} / \mathrm{s}$ in the time of the high and low solar activity;

- correlation between variations of the Earth rotation and earthquake in different time scales decades, interannual and seasonal ones were analysed statistically. Correlation of oscillations with four years period was found.

- anomalies of astronomically determined time and latitude residuals were found before and after the earthquakes around Shanghai, Beijing, Yunan as well as in other stations in the world located close to epicentrums. Four earthquakes were predicted by the use of such anomalies in China. A service of such earthquake prediction was proposed;

the mean acceleration of the Earth rotation rate $w=-62.02 / \mathrm{sec} / \mathrm{cy} / \mathrm{cy}$ was obtained on the basis of old Chinese observations of lunar occultations value $960 \mathrm{AD}$.

Besides mentioned references about 60 papers were published in Chinese journals (with english abstracts) mostly in Annales of Shanghai Observatory and Acta Astronomica Sinica (partly translated into English in Chinese Astronomy and Astrophysics).

University of Texas at Austin, Department of Geological Sciences, Center for Space Research, Institute of Geophysics,Austin, Texas,USA (reported by C.Wilson). Analysis of atmospheric excitation of nonseasonal polar motion have been done by J.Kuehne, S.Johnson and Clark R. Wilson (1993). Analysis of non-seasonal polar motion excitation and atmospheric mass equatorial angular momentum (EAM) over land for the period 1980-89 reveals a clear pattern of high power and correlation during the northern hemisphere $(\mathrm{NH})$ winter followed by low power and correlation during the NH summer. A special case of this pattern occurs for longer than 14 months (from January 1987 to March 1988) when the correlation throughout the NH summer remains statistically significant. During this epoch an average of 72 percent of the non-seasonal polar motion excitation power at frequencies between -30 and +12 cycles per year is linearly related to atmospheric EAM over land. During the SH winter there is significant correlation between the atmospheric EAM over mid-latitude southern ocean and polar motion excitation indicating the existence of a non-equilibrium ocean excitation. The atmospheric excitation power is too small to explain the large correlation during the NH winter. The effects of winds probably account for the deficit in power.

University of Beograd, Mathematical Faculty, Yugoslavia (reported by N.Pejovic) N.Pejovic got a good agreement of the model of the Chandler wobble with modulated frequency dependent amplitude with observations in a nine year period. Agreement is better when influence of groundwater variations is taken into account (Pejovic, 1990, 1992).

Sternwarte der Universitat Bonn, Germany (reported by P.Brosche). P.Brosche in collaboration with oceanographers at Hamburg and geodesists at Bonn, were dealing with theoretical and observational aspects of oceanic influence on the Earth's rotation. Short-period tidal influences have been obtained from hydrodynamical models. The effects predicted in UT have been found in very precise VLBI-data (Brosche, Wünsch, Campbell and Schuh, 1991). The seasonal interaction 
between the atmosphere and the oceans, characterized by varying wind stress and pressure fields, is currently treated by a very complex model. First results and comparisons with LOD and AAM data show that oceanic contribution should not be neglected for a complete balance (Brosche, Wünsch, Frische, Sündermann, Maier-Reimer and Mikolajewicz, 1990).

P.Brosche and J.Wunsch found the periodic variations in the location of the center of mass of the solid Earth due to ocean tides from the ocean tide model of Sailer (1991). The sum of nine partial tides leads to changes in its coordinates $X, Y, Z$ within a range of $1 \mathrm{~cm}$ (Brosche P., and Sündermann J., 1990).

P.Brosche served as a president of the Special Study Group on Long-term Variations in Earth Rotation, of the IAG and was the co-editor of a book on long-term aspects of Earth rotation (Brosche and Wünsch, 1990).

Slovak Technical University, Bratislava, Slovakia (reported by J.Hefty). The comparison of LOD data determined by VLBI, SLR and LLR observations with AAM series from four meteorological data centers carried out by J.Hefty (Hefty 1991a) shows differences in atmospheric series for annual, semiannual, monthly and fortnightly periods. The discrepancies are at $10 \mu \mathrm{s}$ level and are most significant for a semiannual term.

The fortnightly and monthly zonal UT 1 variations were analyzed in order to derive scaling factor $\mathrm{k} / \mathrm{C}$ using optical astrometry, VLBI and SLR data. The frequency dependent $\mathrm{k} / \mathrm{C}$ with "out of phase" constituent, obtained from VLBI and SLR, are consistent with the most recent theories of the Earth's mantle inelasticity and the non-equilibrium ocean tide response (Hefty and Capitaine, 1990).

In the VLBI "intensive" observations on the Westford-Wettzell baseline the UT1 diumal and semidiumal variations caused by non-equilibrium oceans were detected by Hefty (1990). Their interference with nutation errors is discussed. The long term stability of Westford - Wettzell UT1 series was analyzed by Hefty (1993a) showing the systematic effects of quarterly alteration of observing schedule reaching $50 \mu \mathrm{s}$ biases. The analysis of short term variations in GPS polar motion and UT1 series proves the ability of GPS technique to monitor ERP high frequency oscillations for daily and weekly periods. Significant correlation coefficient $(0.4-0.6)$ were obtained from comparison of polar motion and UT1 determined by GPS with those induced by the AAM (Hefty, 1993b).

Observatoire Royal de Belgique, Bruxelles, Belgium (reported by V.Dehant and P.Paquet). New theoretical nutations have been computed and compared to the adopted nutation series, to observations and some other theoretical nutation series by V.Dehant (Dehant, 1992). This model accounts for mantle inelasticity, new core flattening, resonance effect at the periods of the Free Core Nutation (FCN) and the Free Inner Core Nutation (FICN), new rigid nutations and ocean effects (computed from literature). There are remaining discrepancies for which an explanation could be found in atmospheric variations; the atmospheric effects on the nutations have been computed for an oceanless Earth and have been shown to he very efficient (Dehant, Hinderer, Legros and Lefftz, 1993).

Defraigne, Dehant, Hinderer (1993) have used nutation observations jointly with tidal observations in order to determine the FCN period and damping (quality factor). A stacking method applied on both kinds of observations has given a FCN period around 431 days and a quality factor around three thousand.

The amplitudes of the fluctuations observed at 50 and 120 days in the Earth rotation as well as the atmospheric angular momentum, the solar wind, the interplanetary magnetic field were estimated; their mutual correlations and coherencies have been also established by Djurovic and Paquet (Djurovic and Paquet, 1991). The quasi biennial oscillation (QBO), well known in the Earth rotation fluctuations, has been also identified in the solar activity and the atmospheric angular momentum. In the shorter periods (50 and 120 days) a high level of coherency is observed between these signals (Djurovic and Paquet, 1991).

Institute of Astronomy, Academia Romana,Bucuresti, Romania (reported by M.Stavinschi). The long series of astrometric determinations of UTO by the passage instrument have been analysed by M.Stavinschi and collaborators and the star coordinate corrections determined (Ciobanu, Stavinschi, Dinescu, 1992; Stavinschi, Dinescu, Vass, 1992). Theoretical studies of the movement of the Earth instantaneous axis of rotation within the Earth and of the inverse mechanical problem were carried out (Stavinschi, Dinescu, 1992a; Stavinschi 1990; Stavinschi, Mioc, 1993).

Institute of Physical Geodesy,Darmstadt, Germany (reported by E.Groten) E.Groten and H. Yan (Shanghai Observatory) elaborated a "non-rotating" reference frame and its implementation in terms of time. A Departure Point different from Guinot's concept on the moving true equator was studied in detail. It is not assumed to eliminate completely equinox systems. Details of the Departure Point systems with related formulas were published by H.Yan and E.Groten in 1992a, b, c; Groten and Yan, 1993, Yan and Groten, 1993).

Paris Observatory, Paris, France (reported by N.Capitaine). The developments of the celestial pole coordinates have been given as functions of time with an agreement of 0.5 mas with the classical developments of precession and nutation (Capitaine, 1990) and has been applied to the procedure for deriving the Earth Rotation parameters from VLBI (Capitaine, Gontier, 1991). An accurate procedure for deriving UT1 at \pm 0.01 mas accuracy has been developed (Capitaine, 
Gotnier, 1993), based upon the concept of "non-rotating origin" (Guinot, 1979); it has been proposed in the new IERS Standards instead of the usual one and the consequences of its use have been estimated on the practical derivation of UT1. The accurate relationship between the spatial and terrestrial polar motion of the rotation axis and the observed changes of the Celestial Ephemeris Pole have been given (Brzezinski, Capitaine, 1993).

The study of the Earth rotation in the framework of General Relativity has provided the amplitudes of all the post-newtonian nutations in the kinematically non-rotating reference frame (Bizouard et al. 1992). A comparison between recent theories of nutation for a rigid-Earth model (Souchay,1993) shows the large improvement of these new nutation series with respect to those of Kinoshita (1977).

In 1991 the Paris Observatory organized the conference: "Journees 1991 Systemes de Reference Spatio-Temporels - Geodynamique Globale and Systemes de Reference".

GeoForschungs Zentrum, Potsdam, Germany (reported by H.Jochmann and M.Meinig). Investigations on Earth rotation of H.Jochmann, H.Greiner-Mai and their collaborators are related to the influence of geophysical processes on polar motion and the LOD. Especially, excitation functions caused by atmospheric, hydrospheric and core dynamics are discussed.

The relations between the LOD were investigated for different models of fluid motions within the outer core. The rigid rotation of the fluid core, following from the frozen field theory, was extended to the consideration of a non-axial rigid rotation of the Earth's core but it does not improve agreement with variations of the LOD. A better agreement was obtained applying zonal fluid motion of the fluid outer core (Jochmann, 1993).

A further improvement in investigating the relation between time series of geomagnetic field representations and variations of the LOD was obtained by lengthening both time series. Correlations between time series of the geomagnetic field representations were explained assuming that an inner core motion is indicated by the motion of the axis of the geomagnetic dipole. This hypothesis was confirmed by investigating a theoretical model of the relation between inner core rotation and the magnetic dipole axis (Greiner-Mai, 1992a,b; Greiner-Mai, 1993; Greiner-Mai, Felsmann, 1993).

Different periodic variations of the sea level of the Atlantic and Pacific oceans were found, which possibly influence polar motion. The periods of these variations are between 4 and 6 years (Ballani, Felsmann and Jochmann, 1992).

A possible influence of a climate change on Earth rotation was discussed using theoretical models of a climate change. It was found that the variation of atmospheric dynamics caused by climate change has no observable influence on the Earth rotation. A small observable influence on Earth rotation is caused by mass redistributions between the ocean and Antarctic ice sheet (Jochmann, 1992).

Long series of latitude observations together with PZT2 carried out in the Geodetic Institute in Potsdam in the last 100 years have been rereduced by M.Meinig. Linear draft of latitude in this century of $+0.0050^{\prime \prime} \pm 0.0004^{\text {" per year }}$ was obtained against the plate tectonic model AMO-2 values + 0.0005" (Meining, 1991, Meining, 1992, Meining, 1993).

Royal Greenwich Observatory,University of Durham, UK (reported by L.Morrison and R.Stephenson). L.Morrison and R.Stephenson (1992) have completed an analysis of historical observations of solar and lunar eclipses in the period $700 \mathrm{BC}$ to the present with the objective of determining long-term changes in UT and in the length of the day. The data set comprises timed and untimed data from Babylonian, Chinese and European Sources. They have added new material mainly in the period AD 500-1300 since their last study in 1984. These data have helped to define long-term changes in UT on a time scale of millennia (Morrison ans Stephenson, 1991):

1. The average change in the length of the day over the past 2500 years is $+1.7 \mathrm{~ms} /$ century. This is about 25 percent less than the change expected on the basis of tidal friction alone $(+2.3 \mathrm{~ms} /$ century).

2. The length of the day fluctuates between -0.6 and $+4.0 \mathrm{~ms} /$ century about the average trend of $+1.7 \mathrm{~ms} / \mathrm{century}$ on a time scale of 700 years.

Institute of Astronomy, Moscow, Russia (reported by L.Rykhlova). Approximation of the observational data by quasipolynomial model and sero-crossing statistics for signal detection was used for analyses of the ERP and their predictions (Rykhlova et al.1990).

From analysis of 143-year series of the pole coordinates from 1846.0 till 1988.0 (Rykhlova et al. 1991) and the series from 1962.0 till 1982.0 in BIH system (Rykhlova et al. 1992) the following conclusions were obtained: there is a smooth change of the Chandler period within 411-438 days; oscillations with periods of $6.0,8.85,18.6,26.0,32.0,43.3$ and 65.0 years were detected in smoothly changing amplitude.

Analysis of 10-year series of inclination measurements in Finland showed the presence of long-period oscillations with periods similar to those in the pole motion, 0.5 years, 365 days and Chandler ones (Rykhlova et al. 1991). Correlations and interactions of various natural forces with the Earth rotation variations are discussed (Rykhlova 1991) as well as the common properties of the geodynamical and geophysical phenomena (Levitski,Rykhlova 1992, Bobova, Kurbasova, Rykhlova 1993, Rykhlova 1992).

The observations of latitude and longitude variations at Zvenigorod (PZT) and Simeiz (astrolabe) have been carried out. 
Astronomical Institute in Prague, Czech Republic ( reported by J. Vondrak). Atmospheric excitation of polar motion was studied by J.Vondrak (1992) in the interval 1976.5-1988.0. It was found that better agreement between observed and integrated polar motion is obtained if the frequency of the free Chandler wobble is assumed to be variable and amplitudedependent. The method in which the integrated polar path is compared with the observed polar motion is shown to be superior to the one in which the differentiation of the observed polar motion is used to calculate the expected excitations and then compared with the observed ones. The reason for this is the transfer of the noise in the observed data sets and is significant especially for periods shorter than 100 days.

Institute of Geodesy and Geophysies, Wuhang, China (reported by Zhu Yaozhong). Considering the elliptical rotating Earth with inelastic mantle, a fluid core and equilibrium pole tide Zhu Yaozhong determined theoretically the Chandler wobble period and $Q$ factor equal 434.8 days and 71 years respectively. The effects of the inelastic perturbation of the equilibrium pole tide on the Chandler wobble is much larger than the effects of the non-equilibrium part of the pole tide, and mantle inelasticity is likely to be the most important dissipative source of the Chandler wobble. He found that high frequency oscillations of LOD and AAM variations with periods ranging from 40 to 90 days are unstable.

Space Research Centre PAS, Warsaw, Poland (reported by B.Kolaczek). The relations between the EOP reported by the IERS and the dynamical quantities which are coordinates of the Earth's angular velocity vector were studied by A.Brzezinski. The most important result is the derivation of the more rigorous equation of polar motion in which the observed terrestrial coordinates of the Celestial Ephemeris Pole are used as variables instead of the rotation

pole coordinates (Brzeziński A., 1992, Brzezinski and Capitaine, 1993). In view of the sub-diumal resolution of the EOP Brzezinski discussed the problem of distinction between high-frequency polar motion and nutation and finally extended the equation of polar motion for high-frequency variations by including effects of the Free Core Nutation resonance at nearly diurnal retrograde frequency (Brzeziński, 1993).

The short periodical oscillations of polar motion, their predictions and their correlations with such oscillations of the AAM have been analyzed. Variations of the short periodical oscillations of polar motion with periods of 120 , 100 , $80,60,50$ days were analyzed on the basis of SLR and VLBI data. It was found that they are elliptical oscillations with variable amplitudes, ranging 2-5 mas and modulated with the period of about 600 days (Kolaczek 1992a,b; Kosek, Kaczkowski 1992; Kolaczek, Kosek 1992). Correlations of the short periodical oscillations with periods of 80-120 days in polar motion with such oscillations of the AAM variations and the solar activity have been found (Kolaczek and Kosek, 1992).

In early 1988 strong short periodic oscillations with periods of about 35-95 days were detected in both, the $\chi_{y}$ component of polar motion excitation function and LOD variations (Nastula et al. 1990, Nastula 1992). These perturbations are correlated with AAM components variations (Nastula 1992). The $\chi x$ components of atmospheric and geodetic excitation functions of polar motion are not significantly correlated (Nastula 1992). The common periods of about 28 and 40-50 days were detected in $y$ component of both the geodetic and the atmospheric excitation functions of polar motion. These oscillations are influenced mainly by the atmosphere in the northern hemisphere, while the southern influences them only negligibly (Nastula, 1993). The distinct variation of LOD and $\chi_{y}$ components of polar motion excitation function, which occurred in early 1988 seems to be caused by the 40-50 day oscillation in the northern hemisphere atmosphere (Nastula, 1993). The prediction of polar motion coordinates, which now has the accuracy of several milliarcsecond for two weeks, can be improved by taking into account a prediction of short periodic oscillations. The new autocovariance prediction method (Kosek 1992a) or the autoregressive prediction method (Kosek 1992b) were applied to the short periodic oscillations of the polar motion showing that the prediction error depends mostly on the starting prediction moments.

Analyses of amplitude variations of the Chandler and annual wobbles of polar motion in 1846-1988 were carried out on the basis of the longest set of pole coordinates worked out by the Main Astronomical Observatory in Kiev (Nastula et al. 1993). The results show the strong dependence of amplitude modulations of the Chandler wobble on widths of FT filter pass bands. The most energetic oscillations of amplitude variations of the Chandler and annual wobbles of pole coordinates have common periods of about 40, 20-25 and 12-13 years what allows to predict the next deep minimum of the Chandler amplitude for 2010 year(Nastula et al., 1993).

Investigations of the optimal terrestrial reference frame consisting of the minimum number of stations and the highest accuracy were, carried out. Results show that the Primary Terrestrial Reference Frame (PTRF) consisting of 64 SSC collocation points carefully chosen, with a good distribution and accuracy of their coordinates, defines the Terrestrial Reference Frame with almost the same accuracy as the ITRF89 consisting of 119 SSC collocation points (Kosek and Kolaczek, 1991a,b).

Besides these reported above investigations of Earth's rotation more investigations have been carried out especially by the IERS Analyzing Centers. Their results were presented at conferences, mentioned at the begining and can be found also in the papers indicated in the Astronomy and Astrophysics Abstracts. 